\title{
KSM ORATION
}

\section{CAPACITY BUILDING AND INNOVATION IN EHEALTH IN SRI LANKA}

\author{
Vajira H. W. Dissanayake
}

Chair Professor of Anatomy; Director, Human Genetics Unit; Chairperson, Specialty Board in Biomedical Informatics, University of Colombo, Sri Lanka

Corresponding Author: Prof. Vajira H. W. Dissanayake

E.mail - vajira@anat.cmb.ac.lk (i) https://orcid.org/0000-0002-3264-6856

\begin{abstract}
eHealth is the application of the internet and other related technologies in the health care industry. The dramatic improvement in access to the internet from anywhere in the country, the rapid reduction of cost of access, high mobile penetration, and increasing computer literacy has made adoption of eHealth feasible in Sri Lanka. In this background Sri Lanka has made pioneering efforts in human resources capacity development and innovation in the field of eHealth. These developments are summarized in the context of the broader field of eHealth in this oration.
\end{abstract}

Keywords: eHealth, human resources capacity development, innovation

\section{Background}

eHealth is the application of the internet and other related technologies in the healthcare industry to improve access, efficiency, effectiveness and quality of clinical and business processes utilized by healthcare organizations, practitioners, patients and consumers to improve the health status of patients. It is important to note that the emphasis of this definition is on improving the health status of patients.

eHealth can also be seen as healthcare's version of e-commerce or conducting 'health business' electronically. In countries like Sri Lanka, where a free public healthcare service has been the dominant provider of healthcare services for a long time, many find it difficult to perceive the provision of healthcare as a business. In addition when one considers the excellent health related demographics in Sri Lanka, and look at the interventions required to improve it further, investments in information and communication technology does not come high on the list of priorities. On the other hand, when one looks at the information chaos that exists in the healthcare sector and the difficulties in obtaining accurate information in a timely manner, the role that information and communication technology could play to improve the situation is clear. There are many ways in which providers, consumers, and managers within the healthcare system could benefit from the introduction of such technologies.

\section{The Health Informatics Society of Sri Lanka}

It is in this background that the Health Informatics Society of Sri Lanka (HISSL) 
was founded on 15 November 1998. It had its gestation however, in the Medical Informatics Committee of the Sri Lanka Medical Association (SLMA), which was established in January 1997. The HISSL had as its objective the promotion of key benefits of eHealth, such as access to health records at point of care, improved reliability and sharing of records, rapid access to key patient data, timely and supported decision making, improved clinician productivity, reduced errors in diagnosis and treatment, and improved outcomes together with transparency of the health system. Many activities were organized by HISSL to promote these during the first 10 years since its establishment. These included Scientific Congresses in 1999, 2001, 2004, and 2008.

The HISSL also identified the main eHealth challenges that facing Sri Lanka. These challenges related to information inaccurate and incomplete; human resources - inadequate; infrastructure inadequate; quality and security of data and legal issues - not addressed, and financing and sustainability - poor. The HISSL recognized that it had a definite role to play in addressing the challenge of human resource development. To fulfil this need HISSL advocated for the establishment of the MSc in Biomedical Informatics at the Postgraduate Institute of Medicine in the University of Colombo.

\section{MSc in Biomedical informatics at the Postgraduate Institute of Medicine}

Why biomedical informatics and not just eHealth, Health Informatics, or Medical Informatics? Biomedical informatics is the discipline formed by the intersection of computing, medicine and biology. Advances made in the field of genomics and systems biology are finding their way from bench to bedside faster than ever before and transforming medicine in the process. Incorporation of these advances to healthcare is dependent on the availability of information and communication technology infrastructure. Broadening the scope of the course to include biology was done to introduce and prepare the graduates of the course, the new generation of leaders in biomedical and health informatics in Sri Lanka, who would spearhead the development of information and communication technology in the healthcare sector in Sri Lanka to face the challenges of introducing and incorporating these advances to the systems that they would take leadership in developing in the future. The broad subject areas covered in the course are listed below:

- Historical Developments in Computer and Information Technology

- Basic maths and computational logic Computer

Hardware Computer Operating systems and Application Packages

- Data communication and networking Systems analysis and design Database

Systems Programming

- Object oriented systems development Internet and the World Wide Web Web Development Techniques Tools for online communication

- Mobile programming concepts Research Methods and Basic Medical Statistics

- Themes in Biomedical Informatics

- Biomedical data - their acquisition, storage and use Biomedical decision making - probabilistic clinical reasoning Cognitive science and biomedical informatics Essential concepts for biomedical computing Systems design and engineering in heath care Standards in biomedical informatics Natural language and text processing in bioinformatics Imaging and structural Informatics Ethics in biomedical informatics Evaluation 
and Technology Assessment

- Biomedical

Informatics

Applications

- Electronic Health Record Systems and data exchange Management of Information in Healthcare Organisations Consumer health Informatics and Tele-Health Patient care systems Patient monitoring systems Imaging systems in Radiology

- Information retrieval and digital libraries Clinical decisions support systems

- Healthcare financing and information technology

- Telemedicine

- Bioinformatics

- Public Health Informatics

- Informatics in Medical and Health Professional Education

The special feature of this course was that the Ministry of Health gave approval for the course. As a result the Ministry of Health released their medical officers for two years on full pay to follow the course. In addition, they funded the basic course fee of the students. The PGIM in collaboration with the Department of Informatics of the University of Oslo was successful in obtaining further funding from the Norwegian Centre for International Corporation in Higher Education, under its NOMA Grant scheme funded by Norad. These funds made it possible for the students to receive broader international exposure and training in the field of eHealth, which could not be obtained in Sri Lanka.

These graduates today spearhead the development of eHealth in Sri Lanka. Their leadership by itself would be insufficient to make eHealth a success in Sri Lanka. The key to success lies in training other categories of staff in basic information and communication technology skills, as has been recognized previously by others. The future generations of healthcare providers could be prepared for this by incorporating training in information and communication technology skills into medical and other health sciences curricula.

Recommendations made in various international guidance documents could be made use of, for this purpose. In addition those in practice should be afforded the opportunities to develop these skills through continuing professional development programmes.

To date 120 graduates have graduated from the course and they are now spearheading implementation of various eHealth initiatives in the health sector in Sri Lanka. As a result of this, the Ministry of Health has been able to create a sustainable eHealth ecosystem in the health sector in Sri Lanka with minimal dependence on external consultants.

To ensure that Health Informatics becomes a recognized specialty in Medicine the PGIM developed the MD in Health Informatics course with board certification. Today there are $22 \mathrm{MD}$ trainees who would become the first batch of board certified specialists in Health Informatics in the year 2020. This is a significant achievement for Sri Lanka because Sri Lanka is only the second country in the world to recognize Health Informatics as a medical specialty and offer board certification. The only other country to do so is the United States of America, where Clinical Informatics is recognized as a medical subspecialty.

\section{Innovation}

A special feature of the course was the introduction of innovation into the research projects that the MSc students had to carry out in their second year. This has enabled the students to develop some key systems that are today driving the eHealth ecosystem in Sri Lanka. I would like to 
describe two of those systems.

\section{eIMMR - Electronic Indoor Morbidity and Mortality Register}

The collection of nationwide real time data on indoor morbidity and mortality (IMMR) is challenging. The eIMMR system developed by Dr. Buddika Dayaratne and Dr. Nadish Kariyawasam is now making this possible in Sri Lanka.

Initially developed as an MSc student project in 2009 with a grant of Rs. 50,000/from the HISSL, it has now been taken over by the Ministry of Health. It is the first software tool approved by the Ministry of Health for nationwide deployment. As of 14 February 2018 it was implanted in 510 $(82 \%)$ health care institutions (out of a total of 622 secondary and tertiary care institutions). These institutions account for approximately $85 \%$ of the total nationwide hospital admissions in Sri Lanka. There were 1738 user accounts. The database contained over 20 million records. As a result of this system today, there is almost real-time data on morbidity and mortality for the entire country.

\section{DNMS - District Nutrition Monitoring System}

Sri Lanka is seen as a model for delivery of high quality health care in a low resource setting. The nutrition indicators of children however give a different picture. The government of Sri Lanka decided to address this issue with a national action plan coordinated at the highest level of government - the Presidential Secretariat. HISSL was invited to develop the District Nutrition Monitoring System to support the multi-sector action plan for nutrition.

The system consisted of three components. The first component was a smart phone app to be used by public health midwives that enabled them to track and monitor children in their areas. The second component was a web application to enable healthcare managers to monitor the nutrition status of children across the country. The third component alerted different government agencies in the health sector, social services sector, and agriculture sector to take personalised interventions aimed at households that had malnourished children using risk factor data gathered by public health midwives, and enabled them to monitor the effectiveness of the interventions.

The software backbone of the system was DHIS 2. The financial support came from Unicef. The first phase of implementation commenced in June 2016. This involved deploying the system in 3 districts Nuwara Eliya, Matale, and Polonnaruwa. A total of 604 public health midwives were trained and they commenced using the system. A post implementation evaluation and a field level review is currently underway to further strengthen implementation activities.

The Project Team lead by Dr. Roshan Hewapathirana and Dr. Pamod Amarakoon won the award for the best early stage app in South Asia at the mBillionth awards of the Digital Empowerment Foundation in New Delhi, India in July 2016; the Commonwealth Digital Health Awards in October 2016, the eSwabhimani National ICT Awards in November 2016, and the World Summit Award 2016 after having been placed first among entries from 178 countries at the World Summit Awards (WSA) 2016. [https://www.worldsummitawards.org/winn er/district-nutrition-monitoring-systemdnms/]. The WSA has been initiated in 2003 in the framework of the UN World Summit on the Information Society (UN WSIS). It contributes to the UN Sustainable Development Goals agenda by recognizing local digital content with an impact on society, demonstrating the richness and diversity of innovative applications. 


\section{Conclusions}

In conclusion capacity building in eHealth was an absolute necessity if we were to succeed in eHealth in Sri Lanka. By investing in capacity building Sri Lanka has laid the foundation for not only creating human resources capacity for implementation of eHealth in Sri Lanka, but also for sustainable development of the eHealth ecosystem in Sri Lanka without dependence on external experts. The ultimate beneficiaries of these developments would be the people of our country.

\section{Acknowledgements}

I_ would like to acknowledge the contribution of the following individuals and committees to the work and events described in this oration, Prof. Sham Fernando, Prof. Rezvi Sheriff, Prof. Janaka De Silva, Prof. Sundeep Sahay, Dr. Roshan Hewapathiran, Dr. Rohana Marasinghe, Dr. Achala Jayatilleke, Dr. Pandula Siribaddana, members of the Specialty Board in Biomedical Informatics at the Postgraduate Institute of Medicine; Dr. Budhika Dayarathne, Dr. Nadish Kariyawasam, Dr. Pamod Amarakoon and other graduate of the MSc in Biomedical Informatics Course; and the Ministers, the Secretaries, the Director Generals of Health Services, and all other officials of the Ministry of Health from 2007 to date. I would like to acknowledge funding for the work described in here from Norad and Unicef.

\section{References}

1. World Health Organization \& International Telecommunication Union. (2012). National eHealth strategy toolkit. International Telecommunication Union. https//www.who.int/iris/ handle/10665/75211. Accessed 15 June 2018
2. Dissanayake VHW. Developing Human Resources Capacity in Health and Medical Informatics in Sri Lanka. Sri Lanka Journal of Bio-Medical Informatics 2010;1(1):6-11 https://doi.org/10.4038/sljbmi.v1i1.1480

3. Health Informatics Society of Sri Lanka. Digital Health in Sri Lanka: Sustainable Implementation of Digital Health Solutions through Local Capacity Building. Available online at: http://www.hissl.lk/Digital_Health_SL 1998-2017.pdf Accessed 15 June 2018

4. eIMMR: the future of health statistics in Sri Lanka. Kariyawasam NC, Weerasekera BS, Dayaratne MKDRB, Hewapathirana R, Karunapema RPP, Bandara IR. Sri Lanka Journal of BioMedical Informatics. 1, p.S14. https://doi.org/10.4038/sljbmi.v1i0.3549 